\title{
Durable Fire Retardant, Superhydrophobic, Abrasive Resistant and Air/UV Stable Coatings
}

Xiaoyang Wang, ${ }^{1 \#}$ Yao Lu, ${ }^{2 \#}$ Qiaoran Zhang, ${ }^{1}$ Keli Wang, ${ }^{1}$ Claire J. Carmalt, ${ }^{3}$ Ivan P. Parkin, ${ }^{3}$ Zhijun Zhang, ${ }^{1}$ Xia Zhang ${ }^{1,4^{*}}$

${ }^{1}$ National \& Local Joint Engineering Research Center for Applied Technology of Hybrid Nanomaterials, Henan University, Kaifeng 475004, PR China.

${ }^{2}$ Department of Chemistry, School of Biological and Chemical Sciences, Queen Mary University of London, London E1 4NS, UK.

${ }^{3}$ Department of Chemistry, University College London, 20 Gordon Street, London, WCIH OAJ, UK.

${ }^{4}$ State Key Laboratory of Solid Lubrication, Lanzhou Institute of Chemical Physics, Chinese Academy of Sciences, Lanzhou 730000, China

*Corresponding author.Email: zx@licp.cas.cn

\# Equal contribution.

Keywords: Flame-retardant; Superhydrophobic; Non-fluorinated coating

\section{Abstract}

Fabric-based materials such as textiles and papers are widely used in our daily life. However, most of conventional fabrics are highly combustible and easily stained by water and household liquids, susceptible to fire risks and surface contamination/staining. Herein, a non-fluorinated coating that contains the flame-retardants ammonium polyphosphate/pentaerythrotol (APP/PER) and water-repellent silica nanoparticles-polydimethylsiloxane ( $\left.\mathrm{SiO}_{2} @ \mathrm{PDMS}\right)$ is developed. The coated fabric materials prevent fire propagation and are repellant to water, coffee, milk etc. The heat release rate of the $\mathrm{SiO}_{2} @ P D M S / A P P / P E R-c o a t e d ~ c o t t o n$ fabric is 46.33\% lower than that of pure cotton fabric, and the amount of the char yield is increased by 40.4\%. The coatings are durable, resistant to mechanical abrasion and have a long life-time exposure to corrosive liquids and intense UV radiation. The coated fabric materials also exhibit good organic solvent/oil and water separation capability at reduced risks of fire. The facile 
process can be extended to garment and paper industries to lower the fire risks and resist water stains.

\section{Introduction}

Fabric-based materials such as textiles and papers have been widely used in people's daily life ${ }^{[1]}$ However, two major challenges hinder the development robust and durable fabrics. One is that most fabric materials are extremely combustible, which leads to fire hazards in people's daily life and massive costs for fire protection. ${ }^{[2-4]}$ The other challenge is that fabric-based materials, such as textiles and paper, are easily stained by water, spilled tea or coffee. It would greatly benefit the fabric industry, and enhance the lives of the general population if these materials can simultaneously achieve water-proof, UV stable, stain-resistant, fire-resistant and resistant to abrasion.

Flame-retardant treatments on fabric-based materials are usually used to reduce fire hazards, where surface modification is one of the most commonly used methods. ${ }^{[5]}$ Grunlan et al. ${ }^{[6]}$ reported the preparation of flame-retardant coatings on cotton substrates through depositing cycles of polycation/polyanion bilayers. However, these coatings are water-soluble and lose their properties after washing, which greatly hinders their practical applications.

Lotus effect-inspired superhydrophobic surfaces are able to remain dry and clean upon contact with water. ${ }^{[7]}$ Water droplets readily roll off and remove dust from the lotus leaf surfaces so that the surfaces can clean themselves. ${ }^{[8]}$ Superhydrophobic surfaces have shown their stain-resistant and self-cleaning applications on fabric materials. ${ }^{[9]}$ However, the micro and nano-structures that are necessary for superhydrophobicity are inherently fragile and can be 
easily destroyed by external friction, leading to the loss of superhydrophobicity. ${ }^{[10]}$ Upon exposure to UV irradiation, corrosive solutions, and long-term exposure to the environment, such surfaces typically lose their functions, which limits the application. ${ }^{[11-13]}$ In addition, many superhydrophobic coatings are prepared using fluorine-based polymers, which could lead to further environmental issues such as toxicity. ${ }^{[10,14]}$ Therefore, making durable and non-fluorine based superhydrophobic coatings for fabrics is of significant importance for practical applications.

Although flame retardant and superhydrophobic coatings usually were done separately, a few studies have addressed the two challenges in a single material. ${ }^{[4,15]}$ Sun et al. fabricated flame-retardant/self-healing water-proof surfaces on cotton substrates using a dip-coating method. ${ }^{[15]}$ However, the modified coatings contain fluorocarbons, which is expensive and may lead to environmental and safety concerns, especially when such coatings are applied on garments. Furthermore, mechanical and chemical robustness of such flame-retardant and superhydrophobic surfaces have not been well studied to address practical challenges. It remines challenging to make a durable, environmental-friendly and inexpensive coating that has flame retardant properties and water repellence for fabric materials.

Here, a durable and non-fluorinated coating was developed, which has remarkable fire resistance, superhydrophobicity, UV resistance, rubbing resistance and air stability. This coating can be easily treated onto cotton, paper and sponge to stop their combustion, and also repel water, tea, coffee and milk preventing staining. In durability tests showed that the coatings retained their functions after mechanical abrasion, chemical tests and UV/weather 
tests. In addition, a coated filter is able to separate flammable oils/organic solvents and water, and the separation rate is $95 \% \sim 99 \%$ with a reduced risk of fire.

\section{Experimental Section}

Materials and Chemicals. Poly(dimethylsiloxane) vinyl terminated (PDMS, Sylgard 184 kit) and curing agent were purchased from Dow Corning Corporation (USA). Ammonium polyphosphate (APP) and pentaerythrotol (PER) were purchased from Tianjin Kermel Chemical Reagent Company (Tianjin, China). All agents were used as-received without further purification.

Fabrication of hydrophobic $\mathrm{SiO}_{2}$ nanoparticles: $\mathrm{SiO}_{2}$ nanoparticles were treated with hexamethyldisilazane (HMDS) using the methods reported in our previous publication. ${ }^{[16]}$

Flame-resistant/superhydrophobic coating preparation: The sample size of cotton fabric was $30 \mathrm{~mm} \times 40 \mathrm{~mm} \times 3 \mathrm{~mm}$. The cotton fabric was immersed in water solution $(60 \mathrm{~mL})$ containing ammonium polyphosphate (APP) $(50 \mathrm{~g} / \mathrm{L})$ and pentaerythrotol (PER) $(16.7 \mathrm{~g} / \mathrm{L})$ in a water bath at $80{ }^{\circ} \mathrm{C}$ for $1 \mathrm{~h}$, and then dried at $100{ }^{\circ} \mathrm{C}$ for $2 \mathrm{~h}$ to obtain APP/PER coated cotton fabric. Then, the obtained cotton fabric was immersed in ethanol suspension $(100 \mathrm{~mL})$ composed of hydrophobic $\mathrm{SiO}_{2}$ nanoparticles (10 g/L), PDMS (20 g/L) and a curing agent (2 g/L) for $1 \mathrm{~h}$ at $60{ }^{\circ} \mathrm{C}$, followed by curing at $100{ }^{\circ} \mathrm{C}$ for $2 \mathrm{~h}$, then, the $\mathrm{SiO}_{2} @ \mathrm{PDMS} / \mathrm{APP} / \mathrm{PER}$-coated cotton fabric was obtained. The method is equally applicable to other fabric materials such as cotton wool, filter paper and sponge.

Characterization: The surface morphology of the samples was examined using a field emission scanning electron microscope (FESEM, JSM-6701F). The energy dispersive X-ray 
(EDX) analysis was performed on the attachments to the SEM. X-ray photoelectron spectroscopy (XPS, AXISULTRA) was used to confirm the surface chemical composition of the as-prepared sample. Fourier transform infrared (FT-IR) spectra were recorded using a vertex 70 FT-IR spectrophotometer (Bruker Spectrum Instruments, Germany) over 450-4000 $\mathrm{cm}^{-1}$. The contact angles (CA) and sliding angles (SA) of liquids were measured using a DSA-100S optical contact-angle system (Kruss Company, Germany) at ambient temperature. The CAs and SAs of each liquid used a volume of $5 \mu \mathrm{L}$. A digital camera (NIKON, P600) was used to take optical images. Flammability tests were performed on an i-Cone (FTT0402, U. K.), according to ISO5660-1 Standard. The heat flux was $35 \mathrm{~kW} \mathrm{~m}^{-2}$. The size of samples was $100 \mathrm{~mm} \times 100 \mathrm{~mm} \times 1 \mathrm{~mm}$. A RenishawinVia confocal microscope (excitation source: $532 \mathrm{~nm}$ laser) was used to record the Raman scattering spectra of the char residues of the cotton fabric and its composite. Thermogravimetric (TG) measurements were perfomed on a NETZSCH STA449C thermogravimetric analyzer from room temperature to $800{ }^{\circ} \mathrm{C}$ at a rate of $10{ }^{\circ} \mathrm{C}$ $\min ^{-1}$ ( $\mathrm{N}_{2}$ atmosphere). A UV accelerated weathering tester (UV-II, Shanghai Pushen Chemical Machinery; Shanghai, China) was used to evaluate the UV resistance of the samples at $30{ }^{\circ} \mathrm{C}$ and $40 \%$ humidity.

Flame burning tests: (1) Flame burning test. $\mathrm{SiO}_{2} @$ PDMS/APP/PER-coated cotton wool, cotton fabric, filter paper, polyurethane sponge and their respective untreated samples were clamped. Fire produced from methane combustion was applied on these samples for $5 \mathrm{~s}$ and then removed to compare the difference between treated and untreated samples.

(2) Fire tests using a cone calorimeter. In this test, untreated and $\mathrm{SiO}_{2} @ \mathrm{PDMS} / \mathrm{APP} / \mathrm{PER}$-coated cotton fabric samples were used as a representative material, 
these samples were partially wrapped in aluminum foils. The samples were positioned in the sample holder, flattened and then positioned into the load cell together with the sample holder. In the process of ignition, the heat shield was opened to allow the exposed areas of the samples to be radiated by the heat flow of the cone heater (heat radiant power was $35 \mathrm{~kW}$ ), and the pulse igniter was then pointed to the samples to create sparks continuously. When the pyrolyzed flammable gas (methane) was released and contacted with the spark, the samples began to burn. The fire tests ended when the burning of the samples was finished.

Earth repellent tests: Here, the earth repellence refers to the repellence of mechanical abrasion and soil contamination, which were evaluated using two sets of experiments. The first experiment was the sandpaper abrasion test that was used to evaluate the surface durability of the $\mathrm{SiO}_{2} @ \mathrm{PDMS} / \mathrm{APP} / \mathrm{PER}$ coatings. The coated cotton fabric was placed on the sandpaper (grit no. 800-standard glass paper) with $80 \mathrm{~g}$ loading. One abrasion cycle is defined as a $10 \mathrm{~cm}$

abrasion distance in longitudinal and transverse directions, respectively. ${ }^{[17]}$ The second experiment was to dip $\mathrm{SiO}_{2} @ \mathrm{PDMS} / \mathrm{APP} / \mathrm{PER}$-coated samples in mud (a mixture of soil and water at the mass ratio of 1:2) to test their self-cleaning properties. The adhesive forces between mud droplet (droplet size $5 \mu \mathrm{L}$ ) and $\mathrm{SiO}_{2} @$ PDMS/APP/PER-coated samples were also measured using a high-sensitivity micro electromechanical balance system that was reported in our previous work. ${ }^{[18]}$

\section{Results and Discussion}

\section{1. Fire/water resistant surfaces: preparation and characterization}


Figure 1a shows the fabrication process to make a dual functionality fire and water resistant fabric. The cotton fabric material was modified with ammonium polyphosphate/pentaerythrotol (APP/PER) followed by being immersed in $\mathrm{SiO}_{2}$ nanoparticles/polydimethylsiloxane (PDMS) suspensions. Cotton wool, filter paper and polyurethane (PU) sponge were coated by the same process to obtain fire and water resistant properties. The APP particles were coated by taking advantage of the hydrogen-bonding interaction between the $-\mathrm{OH}$ groups in cotton fibers, filter paper and PU sponge and the O-containing groups in APP.

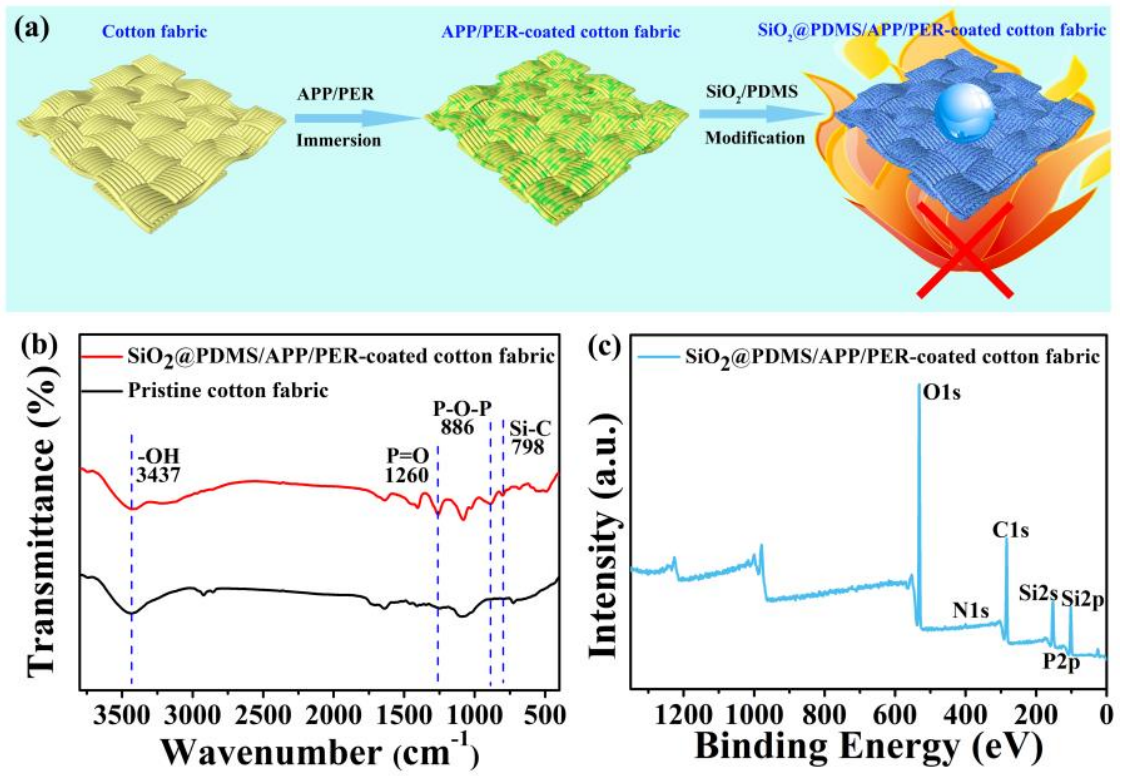

Figure 1 Material synthesis schematic and characterization of surface chemical composition.

(a) Preparation process of a fire and water resistant cotton fabric. (b) FT-IR spectra of the $\mathrm{SiO}_{2} @$ PDMS/APP/PER-coated cotton fabric materials. (c) XPS spectra of the $\mathrm{SiO}_{2} @$ PDMS/APP/PER-coated cotton fabric.

Figure S1 demonstrates the scanning electron microscope (SEM) images of different samples during each preparation process. These samples were smooth at micro scales in their initial stage. After APP/PER treatment, the samples were slightly roughened. When the samples were 
further treated with $\mathrm{SiO}_{2} / \mathrm{PDMS}$ suspensions, their surfaces were significantly roughened creating a dual scale roughness featuring nanostructures. The changes of surface morphologies of cotton wool, cotton fabric, filter paper and PU sponge indicate that this coating method could be generally applied on fabric-based materials. The chemical components on the surfaces of the prepared samples were analyzed by X-ray photoelectron spectroscopy (XPS), Fourier transform infrared spectroscopy (FT-IR), and energy dispersive spectrometer (EDS). In FT-IR spectra (Figure 1b) for pristine cotton fabric, broad $-\mathrm{OH}$ stretch appeared around 3437. However, this $-\mathrm{OH}$ band was notably weakened in the $\mathrm{SiO}_{2} @ \mathrm{PDMS} / \mathrm{APP} / \mathrm{PER}$-coated cotton fabric, compared with the untreated articles and it is observed that new peaks appeared around $1261,860,798 \mathrm{~cm}^{-1}$ (Figure 1b), which correspond to the $\mathrm{P}-\mathrm{O}-\mathrm{P}, \mathrm{P}=\mathrm{O}$ (from APP) and $\mathrm{Si}-\mathrm{C}$ stretches from $\mathrm{SiO}_{2} / \mathrm{PDMS}$, respectively. ${ }^{[5,19]}$ The FT-IR spectra show that the obtained surfaces were efficiently covered with APP, $\mathrm{SiO}_{2}$ and PDMS. XPS and EDS analysis are shown in Figure 1c and Figure S2a (Supporting Information). The C 1s, O 1s, N 1s, P 2p, Si 2s and Si $2 \mathrm{p}$ peaks were observed at 284.8, 532.7, 400.3, 134.2, 150.9, and $102.7 \mathrm{eV}$. The presence of $\mathrm{Si}, \mathrm{N}$ and $\mathrm{P}$ peaks correspond to $\mathrm{SiO}_{2}, \mathrm{PDMS}$, and APP, respectively. ${ }^{[20]}$ The elemental mapping of $\mathrm{C}, \mathrm{N}, \mathrm{O}, \mathrm{Si}$, and $\mathrm{P}$ are demonstrated in Figure S2b-f (Supporting Information); all five elements were uniformly distributed on the surface of the fabric material, indicating that the $\mathrm{SiO}_{2} @ \mathrm{PDMS} / \mathrm{APP} / \mathrm{PER}$ coating was well distributed on the fabric substrate.

\subsection{Burning tests}

Most fabric-basic materials are considered to be highly flammable. ${ }^{[21,22]}$ In order to reduce their flammability and the risk of fire, APP and PER were included in the composite coating due to their highly effective flame-retardant properties. ${ }^{[23,24]}$ To test the fire resistance of the 
$\mathrm{SiO}_{2} @$ PDMS/APP/PER coatings, the untreated and treated cotton wool, cotton fabric, filter paper and PU sponge samples were clamped and exposed to a naked flame for $5 \mathrm{~s}$ as demonstrated in Figure 2 and Video S1. Untreated samples were completed burned, while the treated samples were only slightly charred and did not combust during the test due to their flame resistant properties. To further test the fire resistance of the $\mathrm{SiO}_{2} @ \mathrm{PDMS} / \mathrm{APP} / \mathrm{PER}$-treated samples, the power of fire was increased using a cone calorimeter, which is also able to quantify the heat resistance. Cotton fabric was used as a representative combustible for this test. After being ignited for $26 \mathrm{~s}$, the untreated cotton fabric started to burn, and the fire became stronger between $40 \mathrm{~s}$ and $52 \mathrm{~s}$ (Figure 3a). The untreated cotton fabric was eventually burnt to ashes with a char yield as low as $12.8 \%$. Figure $3 \mathrm{~b}$ shows the combustion process of the $\mathrm{SiO}_{2} @ \mathrm{PDMS} / \mathrm{APP} / \mathrm{PER}$-treated cotton fabric. The treated cotton fabric started charring at $32 \mathrm{~s}$. However, the burning process of the treated sample was slow and its final status was of a graphitized form rather than being turned to ashes. SEM images show that the pure fabric lost its structures (Figure S3a, Supporting Information), whilst the coated fabric maintained its original fibrous structures after the burning test (Figure $\mathrm{S} 3 \mathrm{~b}$, Supporting Information). The char yield of the coated cotton fabric was $40.4 \%$, which is over three times that of the original sample. Figure $3 \mathrm{c}$ shows the heat release rate (pHRR) of the original and the $\mathrm{SiO}_{2} @ \mathrm{PDMS} / \mathrm{APP} / \mathrm{PER}$-coated cotton fabrics, respectively, the latter was much lower than the former during the whole combustion process. The peaks of the pHRR graphs for the original cotton fabric and the $\mathrm{SiO}_{2} @ \mathrm{PDMS} / \mathrm{APP} / \mathrm{PER}$-coated sample were 432.8 and $232.3 \mathrm{~kW} \mathrm{~m}^{-2}$, respectively, indicating that the untreated cotton fabric burned much faster than the coated one. Figure 3d demonstrates the total heat release (THR) for these two samples 
in the process of combustion. The treated cotton fabric released much less heat than the untreated sample, indicating that the $\mathrm{SiO}_{2} @ P D M S / A P P / P E R$ coatings effectively reduced the transformation from chemical to thermal energy and provided good fire protection for the treated cotton fabric.

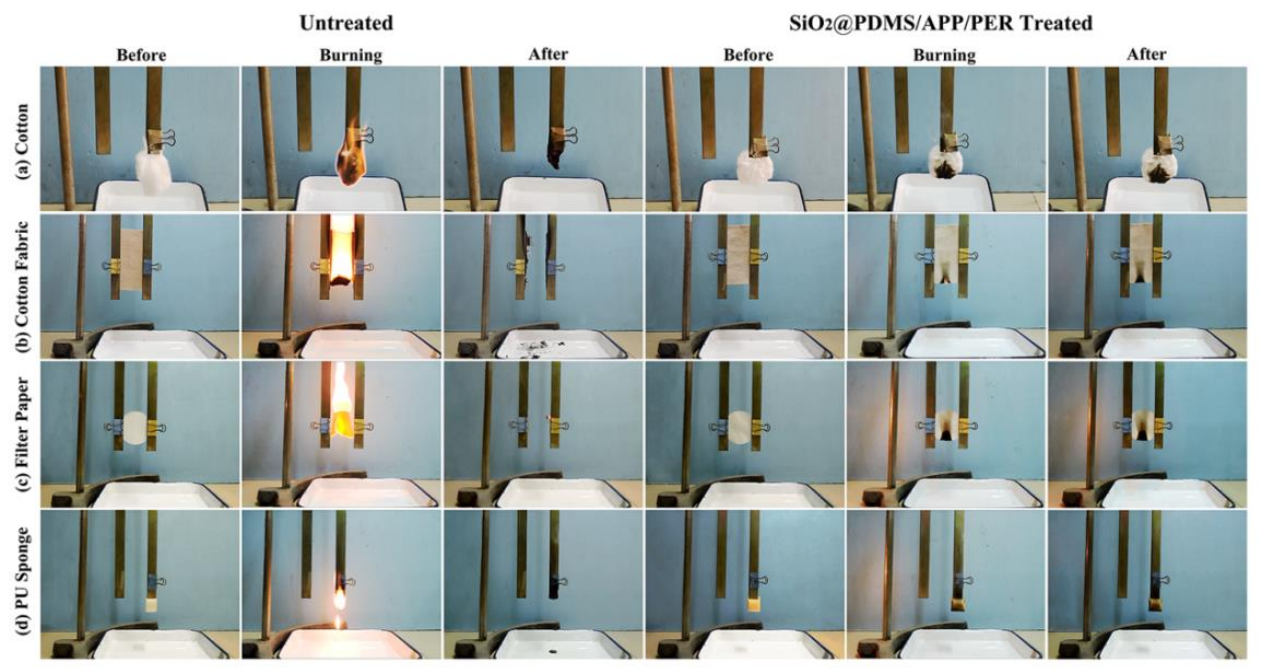

Figure 2 Flame burning tests of samples. Flame burning tests on original and $\mathrm{SiO}_{2} @$ PDMS/APP/PER-coated (a) cotton wool, (b) cotton fabric, (c) filter paper and (d) PU sponge samples. 

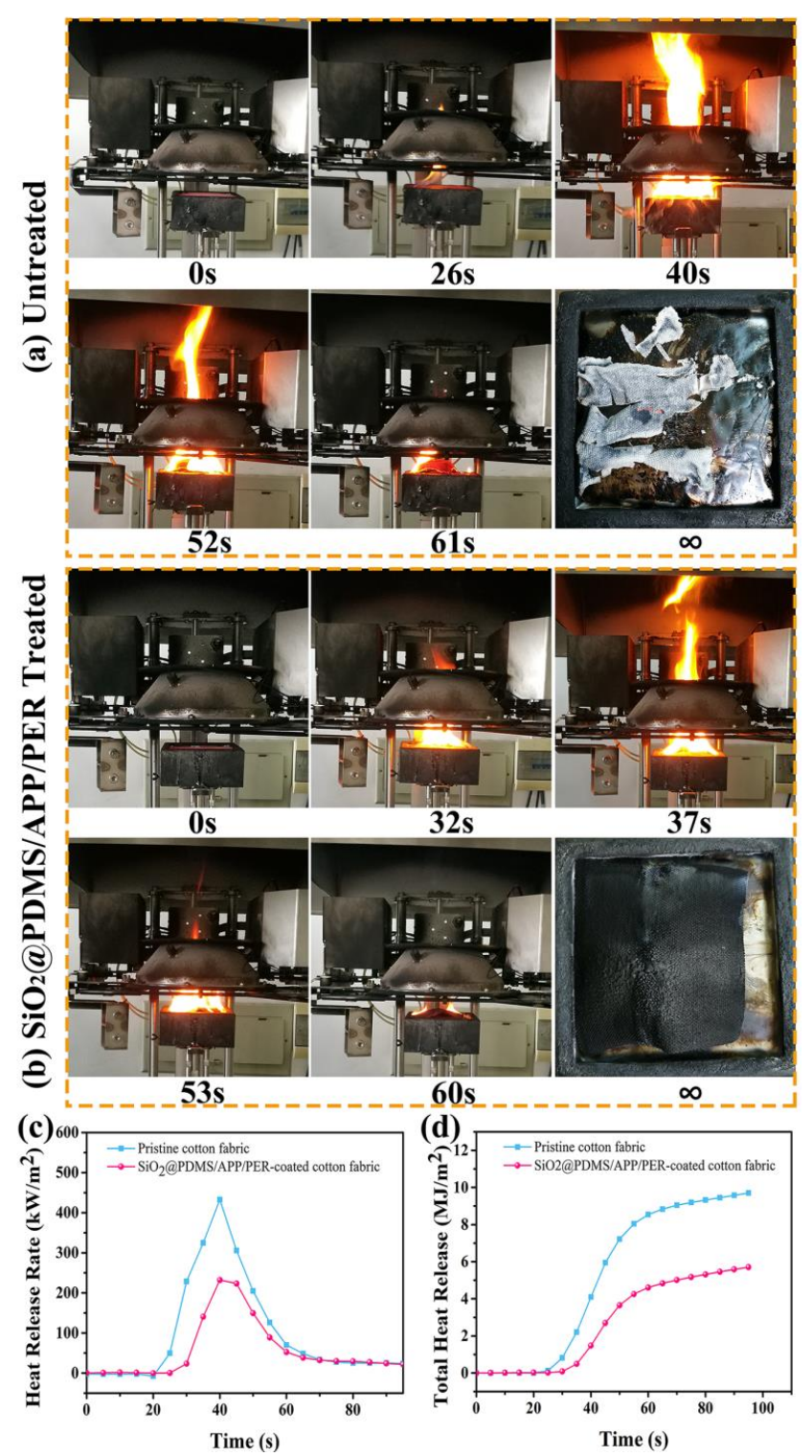

Figure 3 Cone calorimeter tests of cotton fabric before and after $\mathrm{SiO}_{2} @$ PDMS/APP/PER-coating treatment. The combustion process: (a) the original, and (b) the $\mathrm{SiO}_{2} @ \mathrm{PDMS} / \mathrm{APP} / \mathrm{PER}$-coated cotton fabric. (c) Heat release rate, (d) total heat release for both cotton fabrics.

It is generally accepted that "the higher the degree of graphitization, the better heat resistance of char residues due to a barrier effect". ${ }^{[25]}$ To further study the mechanism for the fire resistant property of the $\mathrm{SiO}_{2} @ \mathrm{PDMS} / \mathrm{APP} / \mathrm{PER}$ coatings, the char residue of the untreated and coated 
cotton fabric samples was characterized using Raman spectroscopy to study the degree of graphitization of their carbon residues (Figure 4a and 4b). There are 2 main characteristic peaks in the carbon residue at 1332 and $1585 \mathrm{~cm}^{-1}$, respectively. These peaks corresponded to the carbon atoms vibration from glassy carbons or disordered graphite (D band) as well as graphite carbons ( $\mathrm{G}$ band). ${ }^{[26,27]}$ In addition, the degree of graphitization of the carbon residue was evaluated using the peak area ratio of the $\mathrm{D}$ band and the $\mathrm{G}$ band $\left(\mathrm{I}_{\mathrm{D}} / \mathrm{I}_{\mathrm{G}}\right)$; the higher value of $\mathrm{ID}_{\mathrm{D}} \mathrm{IG}_{\mathrm{G}}$ indicates the lower degree of graphitization. ${ }^{[28]}$ The untreated cotton fabric demonstrated higher value of $\mathrm{ID}_{\mathrm{D}} / \mathrm{I}_{\mathrm{G}}(0.60)$ compared with that of $\mathrm{SiO}_{2} @ \mathrm{PDMS} / \mathrm{APP} / \mathrm{PER}-\mathrm{coated}$ sample (0.518). This indicates that the $\mathrm{SiO}_{2} @ \mathrm{PDMS} / \mathrm{APP} / \mathrm{PER}$ coating is favorable for the chars formation with a higher degree of graphitic and thermo stability. Figure $4 \mathrm{c}$ and $4 \mathrm{~d}$ shows the thermo gravimetric analysis (TGA) and derivative thermo gravimetric (DTG) thermograms of the untreated and the $\mathrm{SiO}_{2} @ \mathrm{PDMS} / \mathrm{APP} / \mathrm{PER}$-coated cotton fabric. The weight of the coated sample started to decrease at $200{ }^{\circ} \mathrm{C}$, followed by a slow decrease from $300{ }^{\circ} \mathrm{C}$ to $400{ }^{\circ} \mathrm{C}$; then its weight decreased dramatically after $400{ }^{\circ} \mathrm{C}$ followed by a steady trend after $500{ }^{\circ} \mathrm{C}$ with a total weight reduction of $71.65 \%$. In terms of the untreated cotton fabric, the initial weight reduction occurred at $300{ }^{\circ} \mathrm{C}$ that was $\sim 100{ }^{\circ} \mathrm{C}$ higher compared with the coated sample. However, the weight of the untreated sample dramatically decreased from $300{ }^{\circ} \mathrm{C}$ to $500{ }^{\circ} \mathrm{C}$ with a total weight reduction of $83.65 \%$, which is higher than the coated sample. Therefore, although the coated sample showed an initial preferential weight reduction, it had a slower weight reduction and a lower final weight loss compared with the untreated sample, which indicates that the $\mathrm{SiO}_{2} @ \mathrm{PDMS} / \mathrm{APP} / \mathrm{PER}$ coating effectively protected the cotton fabric against a rapid 
temperature rise. The mechanism of the fire resistance of the $\mathrm{SiO}_{2} @ \mathrm{PDMS} / \mathrm{APP} / \mathrm{PER}$ coating can be understood as shown in Figure S4. When the $\mathrm{SiO}_{2} @$ PDMS/APP/PER-coated cotton fabric was exposed to fire or high temperature, the catalytic carbonization of APP together with its synergistic agent of PER would lead to cellulose decomposing into graphitized char with low thermal conductivity, ${ }^{[29,30]}$ which blocked the oxygen and heat in the inner polymer during combustion of the cotton fabric. Moreover, APP was able to be involved in radical reactions in gas phase through generating radicals of $\mathrm{PO} \cdot$ during degradation that could quench the radicals of $\mathrm{H} \cdot$ and $\mathrm{OH} \bullet$ in fire and hindered the combustible volatiles evaporation. ${ }^{[31]}$ In addition, $\mathrm{H}_{2} \mathrm{O}$ and $\mathrm{NH}_{3}$ gases generated in the decomposition process could lead to the char layer swelling and diluting the concentration of combustible gas and oxygen. Moreover, inorganic $\mathrm{SiO}_{2}$ nanoparticles could reinforce the layer of chars to prevent their collapse. Therefore, the $\mathrm{SiO}_{2} @ \mathrm{PDMS} / \mathrm{APP} / \mathrm{PER}$ coating imparted excellent fire resistance to its coated materials.

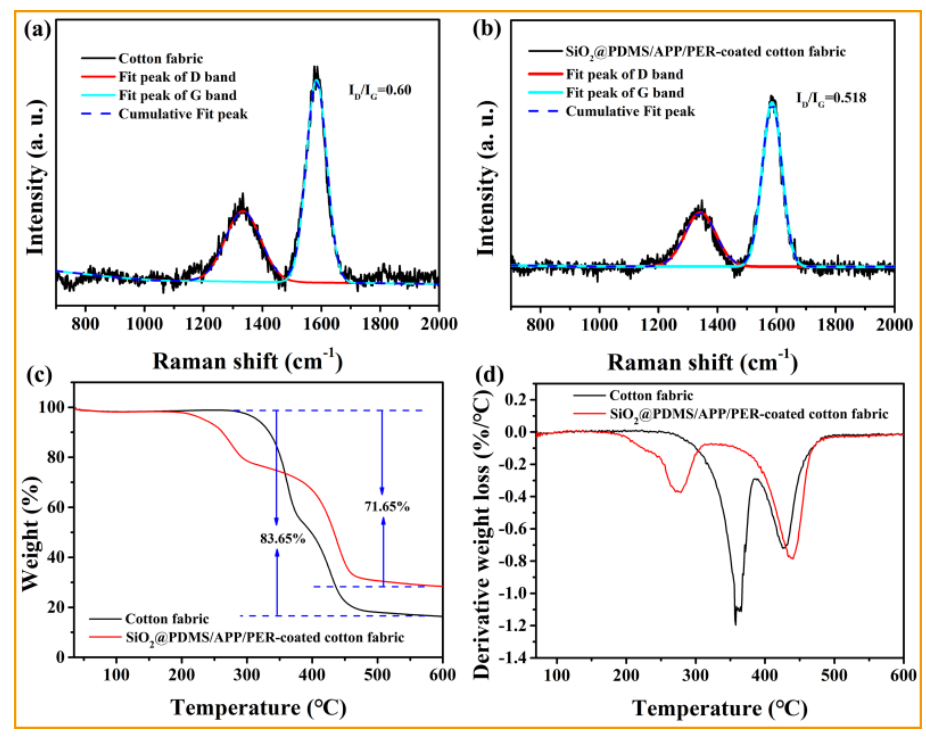

Figure 4 Raman spectra and TGA thermogram. Raman spectra of the char residue: (a) the untreated and (b) the $\mathrm{SiO}_{2} @ P D M S / A P P / P E R-c o a t e d$ cotton fabric materials. (c) TGA and (d) DTG of the untreated and treated samples under a nitrogen atmosphere. 


\subsection{Superhydrophobicity and Stability}

The above results show the fire resistance of the $\mathrm{SiO}_{2} @ P D M S / A P P / P E R$ coating; in this section, superhydrophobicity of this coating is demonstrated. The obtained cotton fabric, filter paper and PU sponge had high repellence to many liquids such as tea, milk, coffee, saturated $\mathrm{NaCl}$ droplet and strong corrosive liquids (Figure 5a-c); all samples have static water contact angles (WCA) more than $150^{\circ}$, and water sliding angles (WSA) less than $8^{\circ}$. Figure $5 \mathrm{~d}$ shows the wettability test of the treated interior PU sponge. The water droplet could not wet the interior PU sponge indicating that superhydrophobicity was obtained throughout the bulk of the treated materials and not confined to the surface. When immersed in water, the obtained cotton wool surface facilitated no detectable water adhesion (Figure 5e-g and Video S2, Supporting Information), indicating the high water repellence. Figure 5h-s shows the processes of water bouncing on treated filter paper; after several more bounces, the droplet completely leaves the surfaces without any stains (Video S3, Supporting Information).

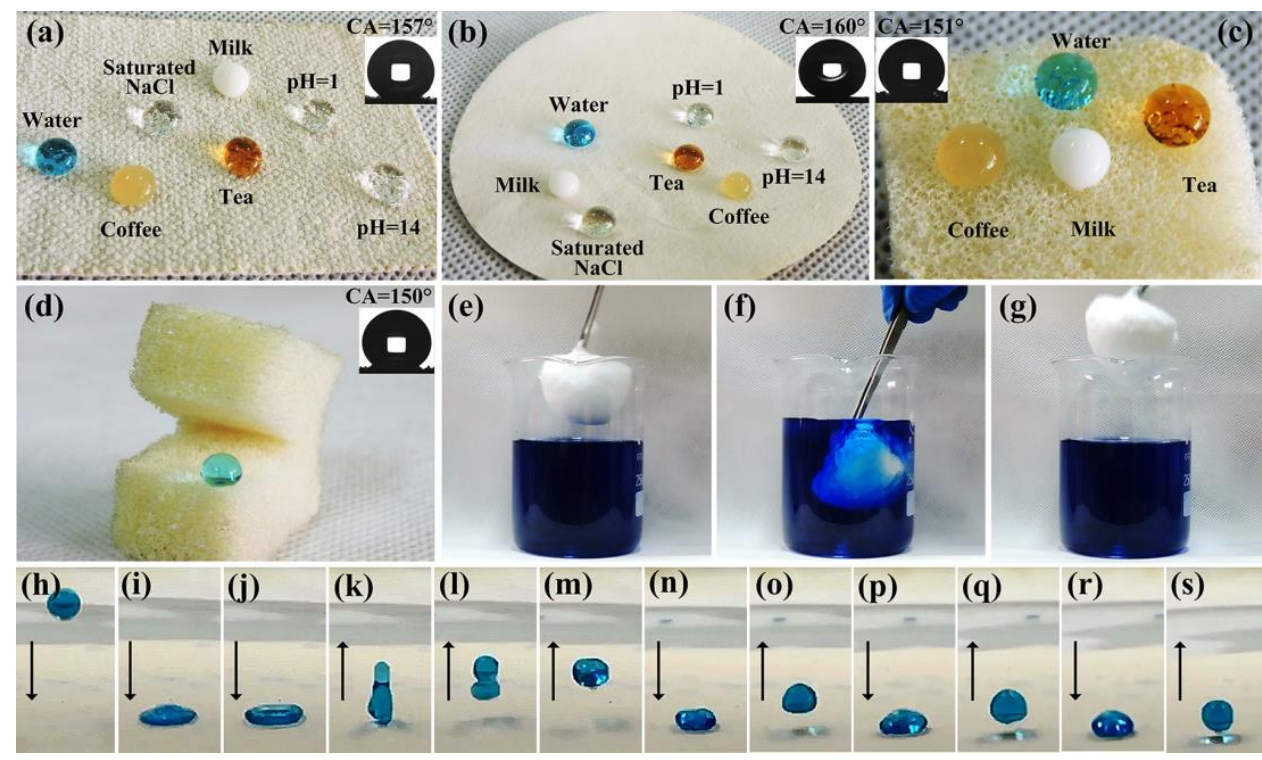

Figure 5 Infiltration analysis of coated samples. Tests of liquid resistance on the $\mathrm{SiO}_{2} @$ PDMS/APP/PER-coated (a) fabric, (b) filter paper, and (c) PU sponge. (d) Interior of 
the PU sponge. (e-g) The treated cotton wool could not be wetted after dipping in water. (Inset: water droplets on respective surfaces). (h-s) Series picture of water bouncing on the coated filter paper.

Earth repellence refers to two items, one is to resist mechanical abrasion and the other is to resist staining and contamination from soil. Mechanical robustness is a key issue for superhydrophobic surfaces. ${ }^{[32,33]}$ The mechanical stability of the fire-resistance cotton fabric was conducted on sandpaper, as shown in Figure 6a. The relationship between the WCA and the abrasion cycle was shown in Figure 6b. After 500 abrasion cycles, the WCAs were maintained above $151^{\circ}$, demonstrating that the coatings had exceptional abrasion resistance. SEM images (Figure 6c) show that surface morphology did not significantly change after 100 cycles of abrasion, compared with that before abrasion. It is believed that the durable micro and nanostructures contribute to the stable superhydrophobicity.

The repellence of soil contamination was evaluated by dipping the coated filter paper and cotton wool into a mixture of soil and water (mud), the coated samples remained dry and clean after being removed from the mud (Figure $6 \mathrm{~d}_{1}-\mathrm{d}_{4}$ and Video $\mathrm{S} 4$ in the Supporting Information), indicating their earth repellence. In contrast, the untreated filter paper and cotton wool were readily wetted and contaminated by mud in the test (Figure S5, Supporting Information). When mud was poured onto the coated surfaces, the mud slid away and the surfaces maintained their clean state (Video S5, Supporting Information), Figure S6 (Supporting Information) shows the adhesive force measurements between mud and the coated samples. The adhesive force for mud and water on the coated filter paper surface were $5.91 \mu \mathrm{N}$ 
and $5.08 \mu \mathrm{N}$, respectively. Whereas the adhesive force for mud and water on the treated cotton fabric material were $7.08 \mu \mathrm{N}$ and $5.93 \mu \mathrm{N}$, respectively. The small adhesive forces for water and mud droplet contribute to the self-cleaning properties and the earth repellence of the coated surface. The adhesive force measurements on untreated filter paper and cotton fabric were not achievable because water and mud were readily adsorbed by the untreated samples once they were contacted.

Importantly, the coated filter paper is mechanically robust and the kneading will not destroy the water-repellency. As demonstrated in Figure 6 $\mathrm{e}_{1-\mathrm{e}_{4}}$ and Video S6, when polluted, water readily removed the dirt from the coated filter paper. The fire-resistant cotton wool also shows mechanical durability, after being kneaded, water droplets still can bounce on the surface, showing extremely water repellence (Figure $6 f_{1-g}$ and Video S7, Supporting Information).

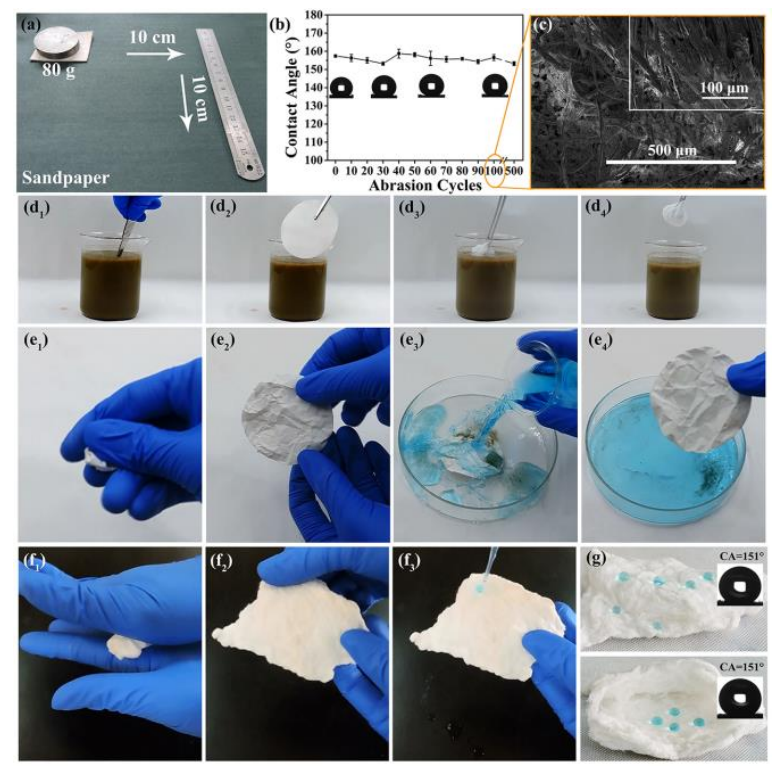

Figure 6 Abrasion and self-cleaning tests. (a) Sandpaper abrasion test. (b) The plot between WCAs and abrasion cycles. (c) SEM image: the $\mathrm{SiO}_{2} @ P D M S / A P P / P E R-t r e a t e d ~ c o t t o n$ fabric surface after 100 abrasion cycles at different magnifications. Self-cleaning of the filter paper and coated cotton $\left(d_{1}-d_{4}\right)$. $\left(f_{1}-f_{3}\right)$ Dirt removal properties of the coated filter paper after being 
kneaded. Water was dyed with methylene blue. (g) Robust superhydrophobic property of the fire-resistant cotton wool.

Air resistance here refers to weather tests that are used to evaluate the coating durability to some outdoor conditions such as wind, temperature, UV radiation, moisture and humidity. Herein, wind test was performed using a hairdryer to evaluate the air resistance of the $\mathrm{SiO}_{2} @$ PDMS/APP/PER-coated surfaces. The treated cotton wool, cotton fabric and filter paper were blown under the wind from the hairdryer for $30 \mathrm{~min}$ as shown in Figure 7 . The treated materials retained their high water repellency with CAs greater than $153^{\circ}$ and SAs smaller than $8^{\circ}$. Figure $7 \mathrm{~b}$ shows the SEM images of the treated materials after wind test and it is clear that the surface micron morphology of these treated materials did not significantly change, resulting that they retained their functional properties.

(a) Optical photo

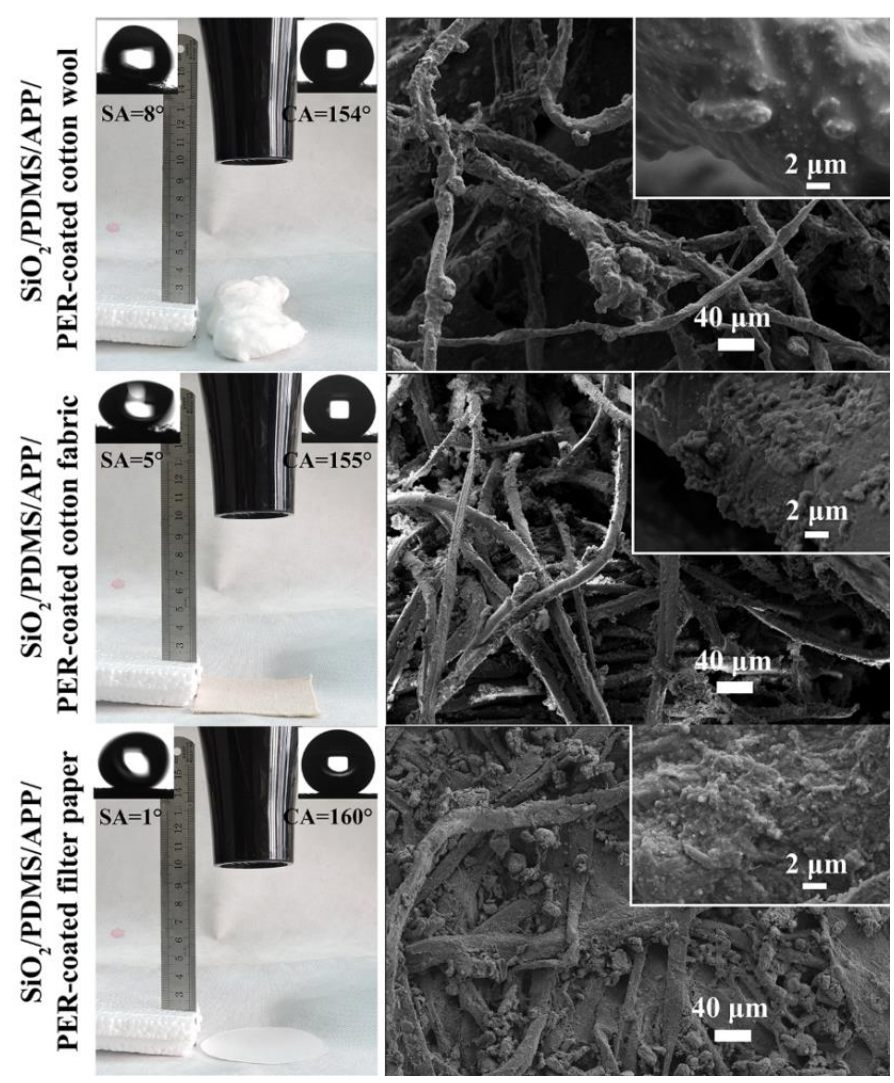


Figure 7 Optical and SEM images of $\mathrm{SiO}_{2} @ \mathrm{PDMS} / \mathrm{APP} / \mathrm{PER}$-coated samples after the wind test. (a) Optical and (b) SEM images of $\mathrm{SiO}_{2} @$ PDMS/APP/PER-coated cotton wool, cotton filter and filter paper after the wind test for $30 \mathrm{~min}$.

Functional coatings are usually degraded due to long-time exposure to high temperature or organic solvents. ${ }^{[34]}$ To further test the coating wetting behavior with temperature, coated cotton fabric materials were heated for $2 \mathrm{~h}$ at different temperatures. There was no significant change in WCAs and WSAs (Figure S7a), indicating that the coating wettability is also stable under high temperatures. Figure S7b-d (Supporting Information) shows that the $\mathrm{SiO}_{2} @ \mathrm{PDMS} / \mathrm{APP} / \mathrm{PER}$-coated cotton fabric samples were immersed in ethanol, acetone and dimethyl sulfoxide (DMSO) for 7 days, respectively, to test their chemical stability with different organic solvents. Every 24 hours, the samples were removed from the solvents and dried at $80^{\circ} \mathrm{C}$ for WCA and WSA measurements. All the samples retained the WCAs of $>150^{\circ}$ and WSAs of $<10^{\circ}$ during the soaking process, indicating that the $\mathrm{SiO}_{2} @$ PDMS/APP/PER coating has good chemical stability.

The $\mathrm{SiO}_{2} @ \mathrm{PDMS} / \mathrm{APP} / \mathrm{PER}-$ coated cotton fabric, filter paper, and PU sponge were positioned under an UV light (wavelength: $313 \mathrm{~nm}$ ) for 7 days. WCAs and WSAs were measured a specific time each day. Figure S7e-g shows that the WCAs and WSAs did not significantly change after prolonged irradiation of UV light for 7 days. All the materials retained the WCA value of $>150^{\circ}$ and WSA value of $<10^{\circ}$ during the process, showing excellent UV resistance.

\section{4. Oil/organic solvents and water separation with reduced risk of fire}


The above results show that the $\mathrm{SiO}_{2} @ \mathrm{PDMS} / \mathrm{APP} / \mathrm{PER}$ coating has remarkable fire and water resistance with high mechanical, chemical and UV stability. One significant application of such coatings is to separate oil/organic solvents and water for the ocean that was contaminated by oil/organic solvents spills. As most of oil is flammable, the material for the separation is required to be fire resistant; the superhydrophobic property of this coating would stop water going through the coated materials and allow the penetration of oil/organic solvents so that oil in water can be separated. Figure S8 and S9 (Supporting Information) show the absorption capacities and separation efficiencies of the $\mathrm{SiO}_{2} @ \mathrm{PDMS} / \mathrm{APP} / \mathrm{PER}$-coated cotton fabric, filter paper and PU sponge to absorb and separate water from various oils/organic solvents such as liquid paraffin, motor oil, cyclohexane, dichloromethane and chloroform. The absorption capacity depends on the form of the materials where the treated PU sponge had the highest absorption capacity of $2039.4 \mathrm{wt} \%$ to $3406.8 \mathrm{wt} \%$ of its own weight. The separation efficiency did not significantly vary from one to another substrate material, and it was $95 \%$ 99\%. The calculation methods of absorption capacity and separation efficiency are described in the supporting information. The results show that the $\mathrm{SiO}_{2} @ P D M S / A P P / P E R-c o a t e d$ fabric materials can be readily used for oil/organic solvent and water separation.
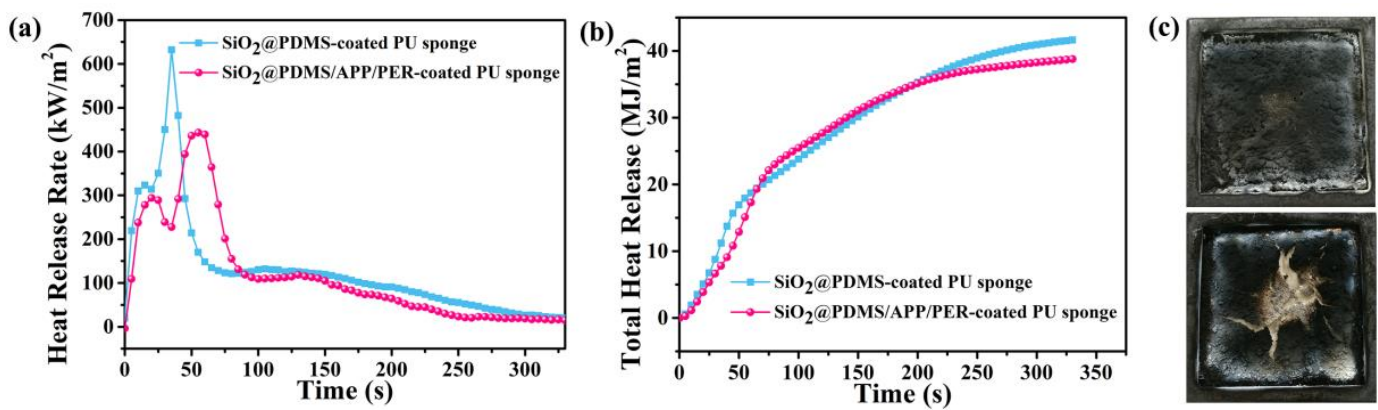

Figure 8 Cone calorimeter tests of PU sponge before and after absorbed with oil. (a) Heat release rate and (b) total heat release of PU sponge before and after treated. (c) Char residues of 
PU sponge treated with $\mathrm{SiO}_{2} @ \mathrm{PDMS}$ (upper) and treated with $\mathrm{SiO}_{2} @ \mathrm{PDMS} / \mathrm{APP} / \mathrm{PER}$ coating (bottom).

To compare the fire resistance with ordinary superhydrophobic oil-water separation materials, a cone calorimetry test was used for evaluating the flame-retardant properties of the material after adsorption of oil. Two pieces of PU sponges with the same mass $(2.47 \mathrm{~g})$ treated with $\mathrm{SiO}_{2} @ \mathrm{PDMS}$ and $\mathrm{SiO}_{2} @ \mathrm{PDMS} / \mathrm{APP} / \mathrm{PER}$ were separately adsorbed with the same quality of motor oil $(8.13 \mathrm{~g})$ for cone calorimetry test and the heat flux was $35 \mathrm{~kW} / \mathrm{m}^{2}$. Figure 8 shows that the $\mathrm{SiO}_{2} @ \mathrm{PDMS}$-coated PU sponge burned rapidly with higher pHRR values, indicating that the superhydrophobic material absorbed oil has flammability characteristics. Compared with the ordinary superhydrophobic PU sponge, the $\mathrm{SiO}_{2} @ \mathrm{PDMS} / \mathrm{APP} / \mathrm{PER}$-coated PU exhibited lower pHRR values and the HRR curve showed a broader peak with the time to reach the peak being delayed by $25 \mathrm{~s}$, implying an enhanced flame-retardant effect. In addition, the THR value of the $\mathrm{SiO}_{2} @ \mathrm{PDMS} / \mathrm{APP} / \mathrm{PER}$-coated PU sponge was smaller than that of the superhydrophobic sample, indicating excellent fire resistance. This is because the APP improved the decomposition of the sponge into a more protective char layer that stopped the heat, oxygen and combustible diffusing into the underlying sponge and the PER as a char forming agent combined with APP further contributed to the formation of excess char layer.

\section{Conclusion}

In conclusion, a non-fluorinated and multiple-functional coating is developed that is strongly resistant against the elemental forces of earth, air, fire and water. The fire resistance and superhydrophobicity provide a unique combination for fabric-based materials (wool cotton, cotton fabric, paper and PU sponge) by integrating flame-retardants ammonium polyphosphate 
and pentaerythrotol into silica nanoparticles-PDMS composite. This coating has remarkable fire resistant properties mainly due to the coating graphitization that stopped the contact between oxygen and substrate materials during combustion. Superhydrophobic properties of the coatings offered protection of water-soluble flame-retardants and enabled superior durability compared with traditional fire-resistant materials. ${ }^{[6]}$ Compared with superhydrophobic materials, ${ }^{[7-10,35,36]}$ our coatings greatly reduced fire risks, particularly for the applications of oil/water separation where superhydrophobic materials are usually exposed to flammable liquids. Table 1 demonstrates a detailed comparison between literature and our work in terms of material functions and durability tests.

The coatings, with their original form of a suspension, provide new concepts for preparation of multifunctional materials using colloids. Our future work will be focused on improving the suspension homogeneity for its long-term storage.

Table 1 Comparison between literature and our work in terms of material functions and durability tests $(\mathrm{Y}=\mathrm{Yes} ; \mathrm{N}=\mathrm{No}$; NA means that the referenced paper did not mention relevant information).

\begin{tabular}{|c|c|c|c|c|}
\hline Reference & Fluorine-free & Superhydrophobic & Fire-resistant & Durability test \\
\hline 2 & $\mathrm{Y}$ & $\mathrm{N}$ & $\mathrm{Y}$ & NA \\
\hline 4 & $\mathrm{Y}$ & $\mathrm{Y}$ & $\mathrm{Y}$ & $\mathrm{NA}$ \\
\hline 5 & $\mathrm{~N}$ & $\mathrm{Y}$ & $\mathrm{Y}$ & $\mathrm{Y}$ \\
\hline 6 & $\mathrm{Y}$ & $\mathrm{N}$ & $\mathrm{Y}$ & $\mathrm{NA}$ \\
\hline 9 & $\mathrm{Y}$ & $\mathrm{Y}$ & $\mathrm{N}$ & $\mathrm{Y}$ \\
\hline 12 & $\mathrm{~N}$ & $\mathrm{Y}$ & $\mathrm{N}$ & $\mathrm{Y}$ \\
\hline
\end{tabular}




\begin{tabular}{|c|c|c|c|c|}
\hline 13 & $\mathrm{~N}$ & $\mathrm{Y}$ & $\mathrm{N}$ & $\mathrm{Y}$ \\
\hline 14 & $\mathrm{~N}$ & $Y$ & $\mathrm{~N}$ & NA \\
\hline 15 & $\mathrm{~N}$ & $Y$ & $\mathrm{Y}$ & $Y$ \\
\hline 16 & $Y$ & $Y$ & $\mathrm{~N}$ & NA \\
\hline 17 & $\mathrm{~N}$ & $\mathrm{Y}$ & $\mathrm{N}$ & Y \\
\hline 18 & Y & $Y$ & $\mathrm{~N}$ & NA \\
\hline 19 & $Y$ & $\mathrm{~N}$ & $\mathrm{Y}$ & NA \\
\hline 20 & $Y$ & $Y$ & $\mathrm{~N}$ & Y \\
\hline 21 & $Y$ & $\mathrm{~N}$ & $\mathrm{Y}$ & NA \\
\hline 22 & $Y$ & $\mathrm{~N}$ & Y & NA \\
\hline 25 & $Y$ & $\mathrm{~N}$ & $\mathrm{Y}$ & NA \\
\hline 29 & $Y$ & $\mathrm{~N}$ & Y & NA \\
\hline 30 & $Y$ & $\mathrm{~N}$ & $\mathrm{Y}$ & NA \\
\hline 31 & $Y$ & $\mathrm{~N}$ & $\mathrm{Y}$ & $Y$ \\
\hline 33 & $\mathrm{~N}$ & Y & $\mathrm{N}$ & Y \\
\hline 35 & $Y$ & $\mathrm{Y}$ & $\mathrm{N}$ & $Y$ \\
\hline 36 & Y & $\mathrm{Y}$ & $\mathrm{N}$ & Y \\
\hline This work & Y & Y & $\mathrm{Y}$ & Y \\
\hline
\end{tabular}

\section{Acknowledgments}

The authors thank Prof. Zuankai Wang for his valuable advice to improve this work. This project is supported by National Natural Science Foundation of China (Grant No.51875173); 
the Innovative Talents Promotion Plan of Henan Province (20HASTIT003) and the West Light Foundation of the Chinese Academy of Sciences. Y. Lu acknowledges the support from QMUL-SBCS start up and the Royal Society Research Grant (RGS\R1\201071).

\section{Author contributions}

$\mathrm{XW}$ and YL contributed equally. YL and $\mathrm{XZ}$ conceived this work. XW, QZ and KW performed the experiments. XW, CJC, IPP, ZZ, YL and XZ analyze the experimental data. YL, $\mathrm{XZ}$ and $\mathrm{XW}$ drafted the manuscript and all authors revised the manuscript.

\section{Conflict of Interest}

The authors declare no conflict of interest.

\section{References}

1 P.J. Rivero, A.Urrutia, J.Goicoechea, F.J. Arregui, Nanomaterials for Functional Textiles and Fibers. Nanoscale Res Lett, 10 (2015) 501-522.

2 Y.C. Li, J. Schulz, S. Mannen, C. Delhom, B. Condon, S.C. Chang, M. Zammarano, J.C. Grunlan, Flame retardant behavior of polyelectrolyte-clay thin film assemblies on cotton fabric. ACS Nano, 4 (2010) 3325-3337.

3 M. Balter, Archaeology. Clothes make the (hu) man Science, 325 (2009) 1329.

4 D.M. Lin, X.R.Zeng, H.Q. Li, X.J. Lai, T.Y. Wu, One-pot fabrication of superhydrophobic and flame-retardant coatings on cotton fabrics via sol-gel reaction. J Colloid Inter Sci, 533 (2019) 198-206.

5 D.M. Lin, X.R. Zeng, H.Q. Li, X.J. Lai, Facile fabrication of superhydrophobic and flame-retardant coatings on cotton fabrics via layer-by-layer assembly. Cellulose, 25 (2018) $3135-3149$. 
6 Y.C. Li, S. Mannen, A.B. Morgan, S.C. Chang, Y.H. Yang, B. Condon, J.C. Grunlan, Intumescent All-Polymer Multilayer Nanocoating Capable of Extinguishing Flame on Fabric. Adv Mater, 23 (2011) 3926-3931.

7 K.S. Liu, L. Jiang, Bio-Inspired Self-Cleaning Surfaces. Annu Rev Mater Res, 42 (2012) 231-263.

8 B. Su, Y. Tian, L. Jiang, Bioinspired Interfaces with Superwettability: From Materials to Chemistry. J Am Chem Soc, 138 (2016) 1727-1748.

9 D.F. Zhi, Y. Lu, S. Sathasivam, I.P. Parkin, X. Zhang, Large-scale fabrication of translucent and repairable superhydrophobic spray coatings with remarkable mechanical, chemical durability and UV resistance. J Mater Chem A, 5 (2017) 10622-10631.

10 Z.L. Chu, S. Seeger, Superamphiphobic surfaces. Chem Soc Rev, 43 (2014) 2784-2798.

11 T.S. Wong, S.H. Kang, S.K.Y. Tang, E.J. Smythe, B.D. Hatton, A. Grinthal, J. Aizenberg, Bioinspired self-repairing slippery surfaces with pressure-stable omniphobicity. Nature, 477 (2011) 443-447.

12 B.C. Li, J.P. Zhang, Durable and self-healing superamphiphobic coatings repellent even to hot liquids. Chem Commun, 52 (2016) 2744-2747.

13 X.C. Tian, S. Shaw, K.R. Lind, L. Cademartiri, Thermal Processing of Silicones for Green, Scalable, and Healable Superhydrophobic Coatings. Adv Mater, 28 (2016) 3677-3682.

14 T.H. Chen, I. Popov, O. Zenasni, O. Daugulis, O.S. Miljanic, Superhydrophobic perfluorinated metal-organic frameworks. Chem Commun, 49 (2013) 6846-6848.

15 S.S. Chen, X. Li, Y. Li, J.Q. Sun, Intumescent flame-retardant and self-healing superhydrophobic coatings on cotton fabric. ACS Nano, 9 (2015) 4070-4076. 
16 X.H. Li, Z. Cao, F. Liu, Z.Z. Zhang, H.X. Dang, A Novel Method of Preparation of Superhydrophobic Nanosilica in Aqueous Solution. Chem Lett, 4 (2006) 14180.

17 Y. Lu, S. Sathasivam, J. Song, C.R. Crick, C.J. Carmalt, P.I. Parkin, Robust self-cleaning surfaces that function when exposed to either air or oil. Science, 347 (2015) 1132-1135.

18 X. Zhang, B. Ding, R. Cheng, S.C. Dixon, Y. Lu, Computational Intelligence-Assisted Understanding of Nature-Inspired Superhydrophobic Behavior. Adv Sci, 5 (2018) 1700520.

19 T. Zhang, H.Q. Yan, M. Peng, L.L. Wang, H.L. Ding, Z.P. Fang, Construction of flame retardant nanocoating on ramie fabric via layer-by-layer assembly of carbon nanotube and ammonium polyphosphate. Nanoscale, 5 (2013) 3013-3021.

20 C.Y. Cao, M.Z. Ge, J.Y. Huang, S.H. Li, S. Deng, S.N. Zhang, Z. Chen, K.Q. Zhang, S.S. Al-Deyab, Y.K. Lai, Robust fluorine-free superhydrophobic PDMS-ormosil@fabrics for highly effective self-cleaning and efficient oil-water separation. J Mater Chem A, 4 (2016) 12179-12187.

21 M.J. Prival, E.C. McCoy, B. Gutter, H.S. Rosendranz, Tris(2, 3-dibromopropyl) phosphate: mutagenicity of a widely used flame retardant. Science, 195 (1977) 76-78.

22 E. Devaux, M. Rochery, S. Bourbigot, Polyurethane/clay and polyurethane/POSS nanocomposites as flame retarded coating for polyester and cotton fabrics. Fire Mater, 26 (2002) 149-154.

23 G.M. Zhou, K. Liu, Y.C. Fan, M.Q. Yuan, B.F. Liu, W. Liu, F.F. Shi, Y.Y. Liu, W. Chen, J. Lopez, D. Zhuo, J. Zhao, Y.C. Tsao, X.Y. Huang, Q.F. Zhang, Y. Cui, An Aqueous Inorganic Polymer Binder for High Performance Lithium-Sulfur Batteries with Flame-Retardant Properties. ACS Central Sci, 4 (2018) 260-267. 
24 S. Bourbigot, M.L. Bras, R. Delobel, R. Decressain, J.P. Amoureux, Synergistic effect of zeolite in an intumescence process: study of the carbonaceous structures using solid-state NMR. J Chem Soc Faraday T, 92 (1996) 149.

25 Q.R. Zhang, Z.W. Li, X.H. Li, L.G. Yu, Z.J. Zhang, Z.S. Wu, Zinc ferrite nanoparticle decorated boron nitride nanosheet: Preparation, magnetic field arrangement, and flame retardancy. Chem Eng J, 356 (2019) 680-692.

26 Y.Y. Dong, Z. Gui, S.H. Jiang, Y. Hu, K.Q. Zhou, Carbonization of Poly(methyl methacrylate) by Incorporating Hydroxyapatite Nanorods during Thermal Degradation. Ind Eng Chem Res, 50 (2011) 10903-10909.

27 Y.B. Hou, W.Z. Hu, L.X. Liu, Z. Gui, Y. Hu, In-situ synthesized CNTs/Bi2 $\mathrm{Se}_{3}$ nanocomposites by a facile wet chemical method and its application for enhancing fire safety of epoxy resin. Compos Sci Technol, 157 (2018) 185-194.

28 M.A. Tamor, W.C. Vassell, Raman “fingerprinting”' of amorphous carbon films. J Appl Phys, 76 (1994) 3823-3830.

29 X. Zhou, S.L. Qiu, W.Y. Xin, C.S.R. Gangireddy, Z. Gui, Y. Hu, Hierarchical Polyphosphazene@Molybdenum Disulfide Hybrid Structure for Enhancing the Flame Retardancy and Mechanical Property of Epoxy Resins. ACS Appl Mater Interfaces, 9 (2017) 29147-29156.

30 J.W. Gu, G.C. Zhang, S.L. Dong, Q.Y. Zhang, J. Kong, Study on preparation and fire-retardant mechanism analysis of intumescent flame-retardant coatings. Surf Coat Tech, 201 (2007) 7835-7841. 
31 X.X. Shi, X.F. Peng, J.Y. Zhu, G.Y. Lin, T.R. Kuang, Synthesis of DOPO-HQ-functionalized graphene oxide as a novel and efficient flame retardant and its application on polylactic acid: Thermal property, flame retardancy, and mechanical performance. J Colloid Interf Sci, 524 (2018) 267-278.

32 W. Barthlott, C. Neinhuis, Purity of the sacred lotus, or escape from contamination in biological surfaces. Planta, 202 (1997) 1-8.

33 D.H. Wang, Q.Q. Sun, M.J. Hokkanen, C.L. Zhang, F.Y. Lin, Q. Liu, S.P. Zhu, T.F. Zhou, Q. Chang, B. He, Q. Zhou, L.Q. Chen, Z.K. Wang, R.H.A. Ras, X. Deng, Design of robust superhydrophobic surfaces. Nature, 582 (2020) 55-59.

34 L.W. Chen, Z.G. Guo, W.M. Liu, Outmatching superhydrophobicity: bio-inspired re-entrant curvature for mighty superamphiphobicity in air. J Mater Chem A, 5 (2017) 14480-14507.

35 N.V. Patil, A.N. Netravali, Bioinspired process using anisotropic silica particles and fatty acid for superhydrophobic cotton fabrics. Cellulose, 27 (2020), 545-559.

36 N.V. Patil, A.N. Netravali, Direct assembly of silica nanospheres on halloysite nanotubes for "Green" ultrahydrophobic cotton fabrics. Adv. Sustain. Syst., 3 (2019), 1900009. 